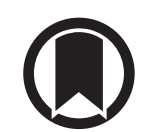

CrossMark

\section{A call for uniformity in implementing the IPAF (interstitial pneumonia with autoimmune features) criteria}

To the Editor:

We commend Oldham et al. [1] for their recent article using the new European Respiratory Society/ American Thoracic Society criteria for interstitial pneumonia with autoimmune features (IPAF) to re-characterise a cohort of interstitial lung disease (ILD) patients in their centre. IPAF defines individuals with interstitial pneumonia and features suggestive of a connective tissue disease (CTD), but not meeting established CTD classification criteria [2]. Of their 422 patients with previously diagnosed idiopathic interstitial pneumonia (IIP) or undifferentiated-CTD ILD (UCTD-ILD), approximately one-third met the IPAF criteria, including nearly $20 \%$ of those with idiopathic pulmonary fibrosis, $25 \%$ with unclassifiable IIP and almost all of those with UCTD-ILD [1]. Importantly, survival was markedly worse in the IPAF group compared with a CTD-ILD cohort [1]. Within the IPAF domains, the presence of at least one clinical criterion was predictive of a favourable outcome, while morphological, multi-compartment involvement carried a significantly increased risk of mortality [1].

Interestingly, only one patient in their IPAF cohort had detectable anti-tRNA synthetase antibodies [1], presumably because patients with myositis-specific antibodies were considered to have CTD-ILD, even in the absence of systemic features of idiopathic inflammatory myopathy (IIM). Physicians often face the challenge of interpreting the significance of a positive autoantibody result in an ILD patient with no systemic manifestations of a CTD, especially when definitive rheumatological criteria have not been established. Such is the case with the IIMs, a group of systemic autoimmune conditions including polymyositis and dermatomyositis (DM). Our awareness of IIMs is critical as ILD occurs in over 50\% of those affected and is the chief determinant of disease progression and prognosis [3].

Since 1970, there have been numerous suggested criteria for the diagnosis and classification of the IIMs [4]. Updated criteria incorporate IIM subgroups based on autoantibody profiles, but there remains no international agreement on how or if such immunoassays should be included $[4,5]$. This variation between definitions can lead to major differences in how a patient with positive myositis antibodies is classified: either as IPAF or CTD-ILD; as demonstrated by OLDHAM et al.'s [1] cohort where such patients were considered to have "anti-synthetase syndrome" and thus CTD-ILD. We believe standardisation of the IIM classification used across our ILD populations is essential, as the inclusion or exclusion of these patients in IPAF cohorts in future studies may have major ramifications for our understanding and further research of IPAF.

Myositis autoantibodies are present in up to $40 \%$ of patients with IIMs and are associated with an increased risk of ILD (table 1) [7, 8]. Anti-tRNA synthetase antibodies are the most commonly detected, and are present in $25-35 \%$ of all IIM patients and up to two-thirds of those who demonstrate ILD [5, 7, 8]. Anti-Jol is the most common of the eight identified anti-synthetase antibodies [7]. The presence of anti-tRNA synthetase antibodies is thought to characterise a unique phenotype, known as "anti-synthetase syndrome", which carries a substantial risk of ILD and increased mortality. Diagnosis remains very challenging due to significant variability in the degree and timing of other features that may include myositis, arthritis, Raynaud's phenomenon, mechanic's hands, skin rashes, sicca syndrome and fever, and ILD can predominate with subclinical myositis in a significant proportion $[5,6]$. Fundamental questions remain unanswered. How do we interpret the presence of auto-antibodies in the absence of other definitive features? What are the implications for management? There is an urgent need to standardise and validate revised definitions of anti-synthetase syndrome, such as that proposed by ConNors et al. [7].

These problems are not just limited to anti-synthetase antibodies. The anti-CADM-140 antibody (also known as anti-MDA5) is associated with "clinically amyopathic DM" and rapidly progressive ILD (CADM-ILD), which has a 6-month survival rate of only 40-55\% [9]. Anti-CADM antibodies have a potential vital role in early identification of CADM-ILD requiring aggressive immunosuppression, but without a clear definition for CADM, research on a uniform cohort remains difficult.

Just as previous research on the patient group now identifiable as IPAF was hindered by inconsistent nomenclature, there is a parallel and urgent need to standardise our approach to the evaluation of CTD in 
TABLE 1 Myositis autoantibodies associated with interstitial lung disease (ILD)

Autoantibody Clinical associations

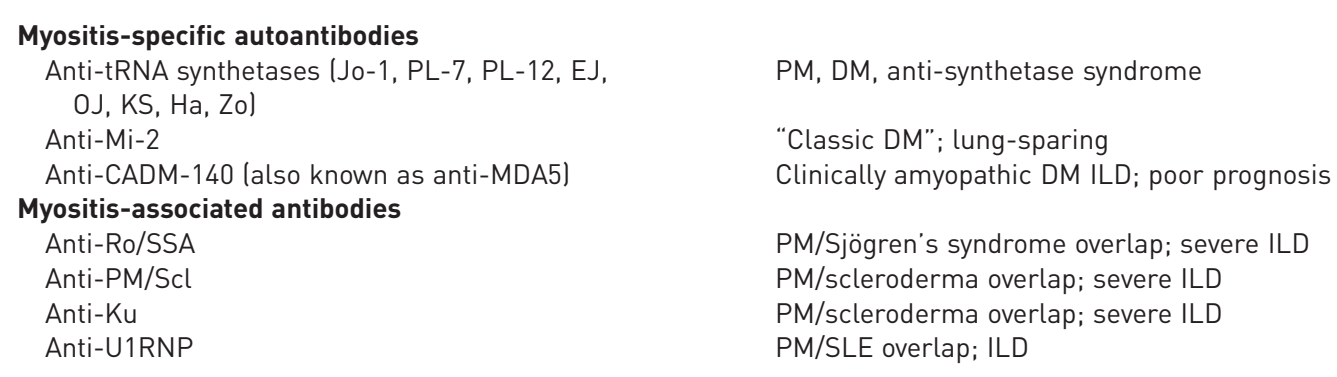

MDA5: melanoma differentiation-associated protein 5; PM: polymyositis; DM: dermatomyositis; SLE: systemic lupus erythematosus. Reproduced and modified from [6] with permission.

our ILD clinics. An added complication is the lack of standardisation of testing and interpretation of autoantibody results. We await with interest the results of the "International Myositis Classification Criteria Project”, a multidisciplinary project launched by the International Myositis Assessment and Clinical Studies Group, sponsored by the National Institute of Environmental Health Sciences of Health and supported by the American College of Rheumatology, European League Against Rheumatism, and The Myositis Association. With enrolment complete and analysis in progress, the goal of the study is to develop consensus classification criteria for the IIMs [10].

We strongly believe there is a need for the ILD community to agree which myositis criteria to use when evaluating our patients. Authors of the IPAF statement recognise that the listed antibodies and serological criteria may require modification as our knowledge evolves [2]. We do not know what the optimum "list" is, but the IPAF classification is a first important step towards developing a minimum dataset that will help determine which criteria should be recommended for clinical practice. If we do not work promptly with rheumatologists, immunologists and internists to standardise our approach to serological testing and rheumatological disease classification, ILD patients with ill-defined extrapulmonary features may continue to fall under different diagnostic umbrellas (i.e. IPAF versus CTD-ILD), which will continue to threaten our ability to make accurate comparisons and conclusions about the natural history and clinical implications of IPAF.

@ERSpublications

There is an urgent need to standardise our approach to connective tissue disease classification in ILD and IPAF http://ow.ly/IPm0304nLVV

Adelle S. Jee ${ }^{1,2}$, Jane F. Bleasel ${ }^{2,3}$, Stephen Adelstein $\oplus^{2,4,6}$, Gregory J. Keir ${ }^{5}$ and Tamera J. Corte ${ }^{1,2}$

${ }^{1}$ Dept of Respiratory and Sleep Medicine, Royal Prince Alfred Hospital, Sydney, Australia. ${ }^{2}$ Sydney Medical School, University of Sydney, Sydney, Australia. ${ }^{3}$ Dept of Rheumatology, Royal Prince Alfred Hospital, Sydney, Australia. ${ }^{4}$ Dept of Clinical Immunology and Allergy, Royal Prince Alfred Hospital, Sydney, Australia. ${ }^{5}$ Dept of Respiratory Medicine, Princess Alexandra Hospital, Brisbane, Australia. ${ }^{6}$ Immunopathology Laboratory, South-west Sydney Pathology Service, Sydney, Australia.

Correspondence: Tamera J. Corte, Dept of Respiratory Medicine, Royal Prince Alfred Hospital, Level 11 Main Building, Missenden Road, Camperdown 2050, Sydney, NSW, Australia. E-mail: tamera.corte@sswahs.nsw.gov.au

Received: June 242016 | Accepted after revision: Aug 172016

Conflict of interest: Disclosures can be found alongside this article at erj.ersjournals.com

\section{References}

1 Oldham JM, Adegunsoye A, Valenzi E, et al. Characterisation of patients with interstitial pneumonia with autoimmune features. Eur Respir J 2016; 47: 1767-1775.

2 Fischer A, Antoniou KM, Brown KK, et al. An official European Respiratory Society/American Thoracic Society research statement: interstitial pneumonia with autoimmune features. Eur Respir J 2015; 46: 976-987.

3 Fathi M, Vikgren J, Boijsen M, et al. Interstitial lung disease in polymyositis and dermatomyositis: longitudinal evaluation by pulmonary function and radiology. Arthritis Rheum 2008; 59: 677-685.

4 Sultan SM, Isenberg DA. Re-classifying myositis. Rheumatology (Oxford) 2010; 49: 831-833.

5 Mahler M, Miller FW, Fritzler MJ. Idiopathic inflammatory myopathies and the anti-synthetase syndrome: a comprehensive review. Autoimmun Rev 2014; 13: 367-371. 
6 Ghirardello A, Borella E, Beggio M, et al. Myositis autoantibodies and clinical phenotypes. Auto Immun Highlights 2014; 5: 69-75.

7 Connors GR, Christopher-Stine L, Oddis CV, et al. Interstitial lung disease associated with the idiopathic inflammatory myopathies: what progress has been made in the past 35 years? Chest 2010; 138: 1464-1474.

8 Lega JC, Reynaud Q, Belot A, et al. Idiopathic inflammatory myopathies and the lung. Eur Respir Rev 2015; 24: 216-238.

9 Ikeda S, Arita M, Morita M, et al. Interstitial lung disease in clinically amyopathic dermatomyositis with and without anti-MDA-5 antibody: to lump or split? BMC Pulm Med 2015; 15: 159.

10 NIEHS. Classification Criteria Study: The International Myositis Classification Criteria Project. www.niehs.nih gov/research/resources/imacs/classificationcriteria/index.cfm Date last accessed: August 7, 2016. Date last updated: March 31, 2015.

Eur Respir J 2016; 48: 1811-1813 | DOI: 10.1183/13993003.01259-2016 ｜ Copyright @ERS 2016

From the authors:

We appreciate the letter of correspondence by A.S. Jee and colleagues highlighting the need to standardise the rheumatologic classification criteria utilised in the diagnosis of a defined connective tissue disease (CTD) when evaluating patients with interstitial lung disease (ILD) for autoimmune features and CTD. This was prompted by our recent publication in the European Respiratory Journal [1]. A.S. Jee and colleagues note that the interstitial pneumonia with autoimmune features (IPAF) cohort in our study contained only one subject with an anti-tRNA synthetase antibody and speculate that patients at our centre with positive antisynthetase antibodies and ILD were diagnosed with an antisynthetase syndrome rather than IPAF [2]. This observation prompted A.S. Jee and colleagues to highlight the numerous criteria that exist for the diagnosis of the idiopathic inflammatory myopathies: polymyositis, dermatomyositis and antisynthetase syndrome and propose that these entities be defined uniformly in the evaluation of patients with ILD. This approach would more precisely distinguish IPAF patients from those with CTD-ILD with potential improvement in the accuracy and prognostic ability of the IPAF criteria.

As noted by A.S. Jee and colleagues, for patients in our ILD registry with an anti-tRNA synthetase antibody, multidisciplinary evaluation yielded a clinical diagnosis of antisynthetase syndrome in all but one patient. An additional patient had evidence of myositis with elevated serum aldolase and creatine kinase levels with a positive anti-Ku antibody, but met IPAF criteria through the clinical (Raynaud's) and morphologic domain (interstitial lymphoid aggregates with germinal centres on surgical lung biopsy) because the anti-Ku antibody is not part of the IPAF criteria. At our institution, our rheumatology colleagues have a heightened awareness of occult CTD presentations in patients with ILD and together we often discern subtle physical findings, such as mild mechanics hands. This more sensitive assignment of a diagnosis of an idiopathic inflammatory myositis in our patients aligns with our practice of aggressively treating ILD in patients with a "myositis" phenotype because of the possibility that ILD in this setting may respond to immunosuppressive treatment and may progress rapidly without such therapy $[3,4]$.

An additional point to consider in the evaluation of the IPAF criteria is centre-specific practice patterns in serologic evaluation. Prior to the publication of the IPAF criteria, we had not routinely sent a full myositis panel on all ILD patients, electing to send only the anti-Jo-1 antibody except in patients where an inflammatory myositis was suspected. Our practice is now evolving with one author (J.M. Oldham) sending a full myositis panel and anti-melanoma differentiation-associated gene 5 antibody in all patients with nonspecific interstitial pneumonitis (NSIP) and the others (M.E. Strek and R. Vij) assessing these antibodies in most ILD patients, even those with usual interstitial pneumonia (UIP) morphology with positive results in a small minority of patients. None of us have yet to incorporate the anti-PM-Scl antibody into our clinical practice, which is an antibody we have not seen our rheumatology colleagues use and is a test that is more difficult for us to perform as it must be sent elsewhere for assay.

Finally, A.S. Jee and colleagues call for uniformity can be interpreted more broadly in the implementation of the rest of the IPAF criteria including decisions about what constitutes diffuse lymphoplasmacytic infiltrates or the presence of multi-compartment thoracic disease [5]. In our study, when determining whether ILD patients met IPAF multi-compartment criteria, we excluded patients with a history of tobacco use from evaluation for intrinsic airways disease, since we could not be certain airway involvement would be "unexplained" in this setting. This decision will need to be revisited as additional centres assess the IPAF criteria in their ILD cohorts.

While the IPAF research criteria are an important first step in identifying features of autoimmunity that might affect prognosis or impact treatment, we believe that there remains great heterogeneity within patients that meet criteria for IPAF, as our study demonstrated. For example, a young African-American 